\title{
Algunas consideraciones sobre el diagnóstico y tratamiento actual del carcinoma prostático organoconfinado*
}

\author{
A. Ipiéns Aznar, S. Napal Lecumberri, I. Pascual Piédrola \\ Servicio de Urología. Hospital de Navarra. Pamplona.
}

Actas Urol Esp 2006; 30 (3): 239-242

$L$ a ciencia se compone de errores que, a su vez, son los pasos hacia la verdad.

\section{Julio Verne}

En los inicios del siglo XXI, el diagnóstico y tratamiento del carcinoma de próstata constituye un capítulo controvertido de la urología cuyas insuficiencias repercuten en la esfera individual, laboral y económica de los individuos y de la sociedad. La posibilidad de padecerlo ocasiona ansiedad quizás excesiva en gran número de varones mayores de 50 años, morbilidad en muchos de los tratados, sobrecarga laboral de incierto beneficio en los sistemas de salud y un costo económico muy elevado para la sociedad en su conjunto.

Sólo en el aspecto científico, un dato evidenciará la complejidad del problema: en el Medline en los últimos doce meses aparecen referenciados 4.928 artículos sobre cáncer de próstata. Este volumen de información es ingente, inalcanzable para cualquier estudioso e indica que el tema es polémico y de actualidad. De los 4.928 artículos, los 100 primeros se distribuyen de la siguiente forma: 25 corresponden a nuevos desarrollos o tratamientos ya conocidos, el mismo número lo hace sobre investigación básica, marcadores, pronóstico, histología y similares, 12 valoran diferentes modalidades de tratamiento, 11 se refieren a técnicas radiológicas de diagnóstico o estadiaje y el resto lo compone una amplia miscelánea que incluye la epidemiología en todas sus áreas junto a artículos de información al paciente y casos anecdóticos.

En los años sesenta y setenta del pasado siglo se vivía respecto al carcinoma de próstata una situación que podría considerarse como de "apatía" o de "resignación", dentro del largo y oscuro túnel que siguió a la euforia inicial causada por los trabajos de Huggins y Hodges en 1941 sobre la hormonosensibilidad de este tumor. La hormonodependencia poco o nada modificó la mortalidad del proceso como pronto se pudo evidenciar, decepción a la que hubo que sumar años después el problema añadido de los efectos cardiovasculares indeseables a largo plazo de la estrogenoterapia.

Esta situación se modificó súbitamente a comienzos de los años ochenta y dio paso a un razonable optimismo en lo que se refiere al diagnóstico precoz y tratamiento del cáncer prostático. El cambio de opinión se debió a dos causas fundamentales: el hallazgo casual, en el curso de estudios inicialmente encaminados a fines legales y forenses, de la existencia en el semen humano de una glucoproteína que acabaría denominándose Antígeno Prostático Específico (PSA) y que Wang et al. popularizarían a partir de 1979, propugnando su cuantificación sérica por su posible valor como marcador de patología prostática en general y específicamente de cáncer cuando las cifras del mismo superaban determinados valores. Tras mejorar al menos aparentemente el problema diagnóstico, el segundo elemento que indujo al optimismo se produjo como consecuencia de la práctica y difusión de la técnica de prostatectomía radical propuesta y comunicada hasta la extenuación por la escuela de Walsh en esos mismos años. Con este bagaje, una sensación de alivio respecto al cáncer de próstata se extendió por los medios urológicos.

*Modificado del Prólogo al libro: Napal S. Evolución Natural del Carcinoma de Próstata Localizado. Pamplona Evidencia Médica, 2005. 
Sin embargo el optimismo inicial no ha ido paralelo con la realidad clínica y en el momento actual el manejo de este proceso en la práctica diaria, al menos conceptualmente, puede calificarse de cualquier manera menos como "capítulo solucionado". Desde el principio y ante la progresiva complejidad del problema, se vio la necesidad de definir conceptos, unificar criterios, valorar diversas conductas e interpretar los resultados terapéuticos. Esto exigió la realización periódica de conferencias de consenso tanto nacionales (L.A. Rioja, Conferencia de Consenso sobre Carcinoma de Próstata, Zaragoza 1992; Castro y Gelabert coordinadores, Documentos de Consenso del Grupo de Expertos AEU), como al desarrollo de Comités Internacionales. Pese a ello persisten diferencias de criterio y actuación muy substanciales.

Por un lado el PSA, aunque sigue siendo por el momento el marcador disponible más potente para el diagnóstico, estadiaje y seguimiento del cáncer de próstata, presenta tremendas limitaciones en su sensibilidad y aún más en su especificidad lo que lo invalida en gran parte como un marcador inicial fiable para el carcinoma de próstata. Hoy día se sabe y acepta que el riesgo de padecer cáncer de próstata existe prácticamente en todos los rangos de PSA (Thompson et al. N Eng J Med 2004; 172: 1297). Aunque una vez mas la opinión no es unánime (Catalona y Loeb, Eur Urol 2005; 48: 541), los datos disponibles sugieren que la determinación periódica, sistemática y seriada del PSA, cuyos rangos de aplicación siguen sin estar estandarizados, seguida por una biopsia prostática cada vez más agresiva, lo único que realmente consigue es aumentar la tasa de diagnósticos, estando sometido a un análisis muy crítico el que dicho proceder aumente el número de curaciones. Aunque un trabajo de Chu et al. (Cancer 2003; 97:1507) atribuye al screening masivo la disminución de la mortalidad por cáncer de próstata apreciada en algunos países que han seguido esta práctica, la opinión dominante no es así y de hecho este mismo año el grupo de Concato (Arch Int Med 2006; 166: 38) llega a la conclusión de que el cribado del cáncer de próstata aumenta su detección precoz pero no reduce el riesgo de fallecer por la enfermedad, ni siquiera en pacientes jóvenes. Respecto a la me- dición del PSA utilizando variables como velocidad de crecimiento, densidad, isoformas y otras, las mismas no aportan mejoras definitivas en los resultados finales terapéuticos, aunque sí una ingente bibliografía al respecto.

Pese a ello el uso indiscriminado del PSA en los varones mayores de 50 años, con honrosas excepciones, constituye prácticamente un "fait accompli". Las razones de ello son muy complejas y variadas e incluyen al propio urólogo y médico generalista (Chesa y Orengo, Arch Esp Urol 2002; 55:113, Cepeda et al. Arch Esp Urol 2005; 58: 403), aunque un análisis en profundidad de este auténtico "fenómeno sociológico" supera las posibilidades de este breve comentario y sólo lo plantea. En Estados Unidos el 80\% de los médicos de familia se controlan anualmente sus cifras de PSA (Barry, BJU 2005; 96: 954) y en los países de nuestro entorno más de la mitad masculina de la población demanda una detección precoz de esta enfermedad, búsqueda que con frecuencia se hace simplemente mediante una determinación aleatoria y fortuita de los niveles de PSA sérico, tal como ha oído vagamente en los medios de comunicación. De esta forma el conflicto está servido.

El uso masivo e indiscriminado del PSA posiblemente sea la causa de que en los últimos cinco años, a diferencia de lo que ocurría hace 20 , en EEUU y posiblemente también en España y en los países en los que esta práctica es habitual, el PSA estaría mas relacionado con la hiperplasia benigna de próstata, su volumen y progresión que con el carcinoma, según un impactante artículo de Stamey et al. (J. Urol 2004; 172: 1297). Este grupo de trabajo, que fue el que inició la "era del PSA" para el diagnóstico a gran escala del cáncer de próstata en 1987 (N England J Med 1987; 317: 909), encuentra ahora que la correlación entre el PSA y el volumen del cáncer prostático ha bajado en los últimos 15 años de 0,7 a 0,1 y refiere que "actualmente, cualquier excusa para biopsiar la glándula prostática tiene una excelente posibilidad edad-dependiente de ser positiva (sic)". El cribado poblacional masivo, al margen de su costo, dudosa eficacia y potencial morbilidad produce una sobredetección de casos que no se hubieran manifestado a lo largo de toda la vida del sujeto pues, entre otras razones, incluye tu- 
mores con una historia natural posiblemente más benigna que los detectados clínicamente o mediante diagnóstico precoz. Además, cabe la posibilidad de la existencia de un tipo de tumores caracterizados por una rápida elevación en la cifras de PSA previas al diagnóstico, lo que implicaría que los mismos no podrían ser conducidos satisfactoriamente ni siquiera mediante el screening (D`Amico et al. N Eng J Med 2004; 351: 125).

Las limitaciones del cribado poblacional junto a las carencias de las determinaciones aisladas del PSA han sido señaladas y estudiadas entre nosotros por el grupo encabezado por A. Berenguer, el cual ha aportado datos muy significativos en este sentido (Berenguer, Practical Cases in Urology, Cribado del Cáncer de próstata, 2004). Pese a estos trabajos y otros similares, no termina de modificarse la opinión dominante, a menudo en ambientes extraurológicos pero con capacidad de toma de decisiones, sobre la bondad del PSA y aceptar el hecho de que esta molécula dista mucho de ser el marcador ideal de la existencia de cáncer prostático que se pensó en un principio, por lo que este hecho debe ser señalado al tiempo que es prioritaria la búsqueda de algún otro procedimiento (sérico o más posiblemente genético) que permita detectar los pacientes afectos de un carcinoma (Hernández et al. Cur Urol ed esp 2005; 4: 112). Además el referido marcador (ideal) debería ser capaz de determinar la capacidad biológica o potencial maligno del mismo (Hessels et al. Rev Urol 2005; 6: 5).

Por si el problema diagnóstico fuera poco, surge el problema añadido de la dificultad existente para valorar correctamente la efectividad del tratamiento radical y agresivo del cáncer de próstata. Esto se debe, como es bien conocido, a la gran prevalencia de cáncer prostático encontrada en la autopsia de los varones mayores de cincuenta años fallecidos por cualquier otra causa y la innegable y significativa menor incidencia de cáncer clínico como causa del fallecimiento, lo que demuestra que no todos los cánceres existentes van a progresar hasta causar la muerte del paciente, por lo que al menos teóricamente, no todos requeririan tratamiento.

Cuando se analizan las escasas series disponibles que valoran la supervivencia de tumores en principio organoconfinados tratados mediante protocolos que incluyen el "ver y esperar", la "vigilancia activa" o la terapia agresiva, los resultados no son concluyentes. Esto puede deberse a que realmente es así o, más posiblemente, a la existencia de algún fallo en los diseños de los diversos estudios, incluyendo el que aún se carezca de la necesaria perspectiva temporal. Albertsen et al. (JAMA 2005; 2093: 2095) refieren que la mortalidad por cáncer de próstata permanece estable a los 15 años de su diagnóstico, por lo que el tratamiento agresivo del cáncer localizado de bajo grado debería ser reconsiderado. Por el contrario y coincidiendo prácticamente en las fechas con el citado artículo, acaba de publicarse el primer trabajo prospectivo y randomizado del grupo de Hill-Axelson et al. (Radical Prostatectomy versus wachful waiting in early prostate cancer, N Eng J Med 2005; 352:1977) que compara los resultados de la actuación agresiva mediante prostatectomía radical y la expectante con un seguimiento medio de 8,2 años en 695 varones con cáncer prostático $\mathrm{T} 1 \mathrm{y} \mathrm{T} 2$, bien o moderadamente diferenciado. El artículo demuestra sin lugar a dudas la superioridad de la actuación agresiva sobre la actitud expectante, pero a este resultado positivo Parker (BJU 2005; 96: 952) hace algunas matizaciones interesantes antes de sacar conclusiones definitivas a favor del primero En primer lugar, según este autor, el tratamiento agresivo no debería ser indicado indiscriminadamente o aceptado como el estándar en todos los casos, máxime en los T1 de grado bajo o medio. La mejoría absoluta en la supervivencia a los 10 años es del 5\%, lo que supone (Barry, ya citado) que se necesitan extirpar 20 próstatas para evitar una muerte a lo largo de 10 años, cifra que podría subir a las 40 en el caso de los tumores detectados mediante screening, considerando el ya comentado posible mejor pronóstico de estos tumores, aspecto este que se investiga, entre otros, en dos trabajos de gran envergadura (PIVOT y PROTECA). Respecto a la disminución en un $10 \%$ de la aparición de metástasis a los 10 años con tratamiento agresivo, puede leerse también como que un $90 \%$ no se va a beneficiar de la actuación terapéutica. A la vista de estos datos la elección del tratamiento por el momento debe ser muy individualizada, 
con gran énfasis en la edad, tipo histológico y velocidad de PSA, además de un adecuado estudio de hechos que incluyen preferencias personales y valoración de años ganados de vida junto a calidad de vida, ya que no puede prescindirse de la morbimortalidad de la terapia agresiva, de todos bien conocida aunque a menudo infravalorada.

Ante estos datos y en un intento, todavía quimérico, de sentar mejor las indicaciones terapéuticas, va ganando terreno el concepto de tumor "insignificante" o "no significativo" (Boccon-Gibod et al. Urology 2005; 48: 895), término aún por definir con exactitud pese a los múltiples intentos realizados hasta la fecha, pero en el que podrían incluirse aquellos tumores diagnosticados en ausencia de clínica mediante biopsia prostática, con buena diferenciación celular y volumen inferior a 0,5 ml. Este es el tipo de tumor encontrado cada vez con mayor frecuencia cuando se aplica una política agresiva de cribado o búsqueda activa de casos con cifras de PSA cada vez mas bajas, al tiempo que se propugna la práctica de las denominadas "biopsias por saturación". Los tumores insignificantes podrían corresponderse con los hallazgos biópsicos de tumor bien diferenciado en un solo cilindro de las 6 ó 10 tomas, o en todo caso en menos del $5 \%$ del total de las muestras. Una actuación agresiva en estos casos posiblemente suponga un sobretratamiento, tal como puede estar ocurriendo entre el 40 y 50\% de los pacientes incluidos en el "European Screening Program" (Draisma et al. J Nat Cáncer Inst 2003; 95: 868) o en un número aún por esclarecer del $94 \%$ de los pacientes con tumor de bajo grado incluidos en el estudio CaPsure (Cancer of the Prostate Strategic Urologic Research Endeavor) que a lo largo del año 2002 recibieron tratamiento radical (Harlan et al. J Urol 2003; 170: 1804). Este proceder explicaría algunos resultados estadísticos hallados, como por ejemplo el aumento de la relación incidencia/mortalidad que en el período de aplicación del programa europeo de screening de próstata pasó de 2.5/1 a $17 / 1$, un incremento de siete veces (Schroder et al. J Urol 2005; 174: 489).

El problema reside en que por el momento no es posible en modo alguno definir estos casos con seguridad y de forma individual y personalizada, independientemente de los criterios clínicos, biológicos o patológicos que se adopten o los modelos matemáticos y estadísticos que se acepten. Hasta disponer de algún medio predictivo fiable del potencial biológico de los tumores prostáticos, los esfuerzos se deben encaminar en los de bajo grado y bajo volumen al desarrollo de protocolos de actuación clínica mediante vigilancia activa (Klotz, J Clin Oncol 2005; 23: 8165), intermedios entre el "ver y esperar" y la actuación invasiva (Richie BJU 2005; 96:951). Esta línea de actuación exige dos premisas esenciales: que sea cómoda y aceptable para el paciente y que no subestime o infravalore los potencialmente agresivos y tributarios de tratamiento radical. Por desgracia, las actuaciones propuestas no reúnen aún ambos requisitos, por lo que dado nuestro nivel de conocimiento actual y posibles avances, salvo la aparición de un hecho nuevo e inesperado, no es previsible un cambio substancial en las indicaciones quirúrgicas actuales de los estadios T1c, T2a y T2b en el futuro inmediato.

Dr. A. Ipiens Aznar

Servicio de Urología. Hospital de Navarra Irunlarrea, s/n - 31008 Pamplona

E-mail: aipiaz@terra.es 